\title{
Quadratic gravity theories in 2+1 dimensions and the topological Chern-Simons term
}

\author{
Antonio Accioly* \\ Instituto de Física Teórica, Universidade Estadual Paulista, Rua Pamplona 145, 01405-000 São Paulo, São Paulo, Brazil
}

(Received 8 April 2003; published 26 June 2003)

\begin{abstract}
The addition of a topological Chern-Simons term to three-dimensional higher-derivative gravity is not a good therapy to cure the nonunitarity of the aforementioned theory. Moreover, $R+R^{2}$ gravity in $(2+1) \mathrm{D}$, which is unitary at the tree level, becomes tree-level nonunitary when it is augmented by the abovementioned topological term. Therefore, unlike what is claimed in the literature, topological higher-derivative gravity in $(2+1) \mathrm{D}$ is not tree-level unitary and neither is topological three-dimensional $R+R^{2}$ gravity.
\end{abstract}

DOI: 10.1103/PhysRevD.67.127502 PACS number(s): 04.50.+h, 02.40.Re, 11.10.Lm, 98.80.Jk

\section{INTRODUCTION}

It is astonishing that a locally trivial and globally nontrivial Einstein-type gravitational theory can be built only by lowering the dimension of our space-time-which is supposed to be four dimensional—by one unit. In fact, threedimensional general relativity is dynamically trivial: outside sources, spacetime is flat-all effects of the localized sources are on the global geometry, which is fixed by the singularities of the worldlines of the particles [1]. The quantum mechanical analogue of this triviality emerges when the HilbertEinstein action related to this theory is quantized. It is easy to establish that the theory does not possess any propagating degrees of freedom. In other words, there are no gravitons. The preceding considerations lead us to raise the following important question: Is it possible to build a nontrivial generally covariant three-dimensional gravity theory having propagating degrees of freedom? The answer is affirmative. Actually, this can be done at least in two different ways:

(i) Adding a topological nontrivial term to general relativity in $(2+1) \mathrm{D}$ [2]. Ordinary Einstein gravity which is trivial acquires now a propagating, massive, spin-2 mode. This theory is ghost-free and causal, although of the thirdderivative order.

(ii) Including the four-derivatives terms $\int R^{2} \sqrt{g} d^{3} x$ and $\int R_{\mu \nu}^{2} \sqrt{g} d^{3} x$ into three-dimensional Einstein action [3-5]. In this case we have a class of effectively multimass models of gravity of fourth-derivative order. In addition to the massless excitations of the field which, incidentally, are nonpropagating such as in three-dimensional gravity, there are now-for the general amount of the two new terms - massive spin-2 and massive scalar excitations. We review in Sec. II the main properties of this theory.

On the other hand, unlike topological gravity in $(2+1) \mathrm{D}$, three-dimensional higher-derivative gravity (3DHDG) is nonunitary at the tree level. How can we remedy this situation? It seems, at first sight, that we should add a topological Chern-Simons term to 3DHDG, since Einstein gravity augmented by a topological term is unitary at the tree level [6]. But, as we shall show in Sec. III, this artifice does not cure the tree level nonunitarity of 3DHDG. Consequently, unlike what is claimed in the literature [7], 3DHDG augmented by a

\footnotetext{
*Electronic address: accioly@ift.unesp.br
}

Chern-Simons term is nonunitary at the tree level.

We conclude in Sec. IV by proving that topological $R$ $+R^{2}$ gravity in $(2+1) \mathrm{D}$ is also nonunitary at the tree level-an interesting and at the same time curious fact, since $R+R^{2}$ gravity in $(2+1) \mathrm{D}$ is tree-level unitary. This amazing result does not simply oppose the claim in the literature [7]; it is against what, in a sense, one should intuitively expect. We employ natural units in which $c=\hbar=1$. Our conventions are to use a metric with signature $(+--)$ and define the Riemann and Ricci tensors as $R_{\lambda \mu \nu}^{\rho}=-\partial_{\nu} \Gamma_{\lambda \mu}^{\rho}+\partial_{\mu} \Gamma_{\lambda \nu}^{\rho}$ $-\Gamma_{\lambda \mu}^{\sigma} \Gamma_{\sigma \nu}^{\rho}+\Gamma_{\lambda \nu}^{\sigma} \Gamma_{\sigma \mu}^{\rho}$ and $R_{\mu \nu}=R_{\mu \nu \rho}^{\rho}$.

\section{THREE-DIMENSIONAL HIGHER-DERIVATIVE GRAVITY}

3DHDG is defined by the action [3-5]

$$
S=\int d^{3} x \sqrt{g}\left[\frac{2 R}{\kappa^{2}}+\frac{\alpha}{2} R^{2}+\frac{\beta}{2} R_{\mu \nu}^{2}-\mathcal{L}_{M}\right]
$$

where $\mathcal{L}_{M}$ is the Lagrangian density for matter and $\alpha$ and $\beta$ are constants with dimension $L$. Here $\kappa^{2} \sim L$ is not necessarily related to the Einstein constant in $(3+1) \mathrm{D}$. In the linear approximation, i.e. $g_{\mu \nu}=\eta_{\mu \nu}+\kappa h_{\mu \nu}$, and in a threedimensional version of the Teyssandier gauge $[8,9]$, the original field equations, namely,

$$
\begin{aligned}
& \left(2 / \kappa^{2}\right) G_{\mu \nu}+\frac{1}{2} \alpha\left[-\frac{1}{2} g_{\mu \nu} R^{2}+2 R R_{\mu \nu}+2 \nabla_{\mu} \nabla_{\nu} R\right. \\
& \left.\quad-2 g_{\mu \nu} \square R\right]+\frac{1}{2} \beta\left[-\frac{1}{2} g_{\mu \nu} R_{\rho \sigma}^{2}+\nabla_{\mu} \nabla_{\nu} R+2 R_{\mu \rho \lambda \nu} R^{\rho \lambda}\right. \\
& \left.\quad-\frac{1}{2} g_{\mu \nu} \square R-\square R_{\mu \nu}\right]+\frac{1}{2} T_{\mu \nu}=0,
\end{aligned}
$$

reduce to

$$
\begin{aligned}
& \left(1-\frac{1}{4} \beta \kappa^{2} \square\right)\left[-\frac{1}{2} \square h_{\mu \nu}+\frac{1}{4} \eta_{\mu \nu} \bar{R}\right]+\frac{1}{2}\left(\Gamma_{\mu, \nu}+\Gamma_{\nu, \mu}\right) \\
& =\frac{1}{4} \kappa\left(T_{\mu \nu}-\eta_{\mu \nu} T / 2\right), \\
& 0=\Gamma_{\mu} \equiv\left(1-\frac{1}{4} \beta \kappa^{2} \square\right) \gamma_{\mu \lambda}^{\lambda}-\left(\alpha+\frac{1}{2} \beta\right) \frac{1}{2} \kappa^{2} R_{, \mu},
\end{aligned}
$$


where $\bar{R} \equiv \frac{1}{2} \square h-\gamma_{, \mu \nu}^{\mu \nu}$ and $\gamma_{\mu \nu} \equiv h_{\mu \nu}-\frac{1}{2} \eta_{\mu \nu} h$. The solution of the preceding system of equations is given by $h_{\mu \nu}=h_{\mu \nu}^{(E)}$ $+\psi_{\mu \nu}-\Phi \eta_{\mu \nu}$, where $h_{\mu \nu}^{(E)}, \psi_{\mu \nu}$ and $\Phi$ satisfy the following equations:

$$
\begin{gathered}
\square h_{\mu \nu}^{(E)}=\frac{1}{2} \kappa\left[T \eta_{\mu \nu}-T_{\mu \nu}\right], \quad \gamma_{\mu \nu}^{(E), \nu}=0, \\
\gamma_{\mu \nu}^{(E)}=h_{\mu \nu}^{(E)}-\frac{1}{2} \eta_{\mu \nu} h^{(E)} ; \\
\left(\square+m_{1}^{2}\right) \psi_{\mu \nu}=\frac{1}{2} \kappa\left[T_{\mu \nu}-\eta_{\mu \nu} T / 2\right], \quad \psi_{\mu \nu}{ }^{\mu \nu}-\square \psi=0 ; \\
\left(\square+m_{0}^{2} / 2\right) \Phi=\kappa T / 4 .
\end{gathered}
$$

Here $m_{0}^{2} \equiv 1 / \kappa^{2}\left(\frac{3}{4} \beta+2 \alpha\right)$ and $m_{1}^{2} \equiv-4 / \beta \kappa^{2}$. Using the equations above we find that the metric for an isolated static particle source of mass $m$ located at $\mathbf{r}=\mathbf{0}$ is

$$
\begin{aligned}
& h_{00}=\frac{\kappa m}{8 \pi}\left[\kappa_{0}\left(m_{1} r\right)-\kappa_{0}\left(m_{0} r\right)\right], \\
& h_{11}=h_{22}=\frac{\kappa m}{8 \pi}\left[2 \ln r+\kappa_{0}\left(m_{1} r\right)+\kappa_{0}\left(m_{0} r\right)\right],
\end{aligned}
$$

where $\kappa_{0}$ is the modified Bessel function of the order of zero and $m_{0}^{2}$ and $m_{1}^{2}$ are assumed thereafter to be real in order to avoid the presence of tachyonic particles in the dynamical field. This metric greatly resembles, mutatis mutantis, the four-dimension metric of a straight $U(1)$ gauge cosmic string in the context of linearized gravity found by Linet and Teyssandier [10]. In addition, as $m_{0}$ and $m_{1} \rightarrow \infty$ it tends to the solution of the linearized version of three-dimensional Einstein equation in the de Donder gauge found by Deser [11].

The nonrelativistic potential is of course given by

$$
V(r)=2 \bar{G} m\left[\kappa_{0}\left(m_{1} r\right)-\kappa_{0}\left(m_{0} r\right)\right],
$$

where $\bar{G} \equiv \kappa^{2} / 32 \pi$. Note that $V(r)$ behaves as $2 \bar{G} m \ln \left(m_{0} / m_{1}\right)$ at the origin and as

$$
2 \bar{G} m\left[\sqrt{\frac{\pi}{2 m_{1} r}} e^{-m_{1} r}-\sqrt{\frac{\pi}{m_{0} r}} e^{-m_{0} r}\right]
$$

asymptotically.

Three comments are in order here:

(i) Unlike the Newtonian potential in $(2+1) \mathrm{D}, V_{N}$ $=2 \bar{G} m \ln \left(r_{0} / r\right)$, which has a logarithmic singularity at the origin and is unbounded at infinity, the potential concerning 3DHDG is extremely well behaved: it is finite at the origin and zero at infinity.

(ii) $V(r) \rightarrow 0$ as $m_{0}$ and $m_{1} \rightarrow \infty$, confirming in this way the well known fact that the standard correspondence of three-dimensional Einstein theory with Newton theory breaks down [12].

(iii) The nonrelativistic potential can also be computedwithout appealing to the solution of the field equationsusing an algorithm developed in Ref. [5].
We discuss now, in passing, two interesting effects of the metric related to an isolated static point particle of mass $m$ : the existence of a gravitational force on a slowly moving test particle and the gravitational deflection of light rays.

Gravitational acceleration. The gravitational acceleration of a slowly moving test particle of mass $m$ in the gravitational field in hand is

$$
\gamma^{k}=-2 \bar{G} m\left(x^{k} / r\right)\left[m_{0} \kappa_{1}\left(m_{0} r\right)-m_{1} \kappa_{1}\left(m_{1} r\right)\right] .
$$

Therefore, the gravitational force related to (1) is everywhere attractive if $m_{0}<m_{1}$, is repulsive if $m_{0}>m_{1}$ (antigravity) and vanishes if $m_{0}=m_{1}$ (gravitational shielding). Note that this force does not exist in general relativity in $(2+1) \mathrm{D}$. It is peculiar to 3DHDG.

Light bending. For a light ray in the $x-y$ plane, coming from infinity with an impact parameter $b$, the deflection angle is given by

$$
\theta=4 \pi m \bar{G}\left[1-e^{-m_{1} / b}\right] .
$$

It is interesting to note that the harmless massive scalar mode does not contribute anything to the light deflection. This can be easily explained if we take into account that $R+R^{2}$ gravity in $(2+1) \mathrm{D}$ is conformally related to three-dimensional Einstein gravity.

Despite these nice properties, 3DHDG is nonunitary at the tree level; nevertheless, it is tree-level causal.

\section{TREE-LEVEL NONUNITARITY OF TOPOLOGICAL THREE-DIMENSIONAL HIGHER-DERIVATIVE GRAVITY}

In the hope of curing the tree-level nonunitarity of 3DHDG we add a Chern-Simons term to this theory. The resulting Lagrangian can be written as

$$
\mathcal{L}=-\frac{2 R \sqrt{g}}{\kappa^{2}}+\left(\frac{\alpha}{2} R^{2}+\frac{\beta}{2} R_{\mu \nu}^{2}\right) \sqrt{g}+\mathcal{L}_{\text {C.S. }},
$$

where

$$
\begin{aligned}
\mathcal{L}_{\text {C.S. }} & =(1 / 2 \mu) \varepsilon^{\mu \nu \lambda}\left(R_{\beta \mu \nu}^{\alpha} \Gamma_{\alpha \lambda}^{\beta}-\frac{2}{3} \Gamma_{\beta \mu}^{\alpha} \Gamma_{\gamma \nu}^{\beta} \Gamma_{\alpha \lambda}^{\gamma}\right) \\
& =\left(\varepsilon^{\lambda \mu \nu} / \mu\right) \Gamma^{\rho}{ }_{\sigma \lambda}\left(\partial_{\mu} \Gamma_{\rho \nu}^{\sigma}+\frac{2}{3} \Gamma_{\omega \mu}^{\sigma} \Gamma_{\nu \rho}^{\omega}\right)
\end{aligned}
$$

and $\mu$ is a dimensionless parameter.

In order to probe the tree-level unitarity of the aforementioned theory we couple the propagator, $\mathcal{O}$, to external conserved currents, $T^{\mu \nu}$, compatible with its symmetries, i.e. we cast the amplitude in the form

$$
\mathcal{A}=\mathrm{g}^{2} T^{\mu \nu} \mathcal{O}_{\mu \nu, \rho \sigma} T^{\rho \sigma},
$$

where $g$ is the effective coupling constant of the theory, and afterward we examine the current-current amplitude at the poles [13]. To accomplish this we have to find the propagator, which involves much algebra. However, the calculations can be greatly simplified if we make use of a class of operators made up by the usual three-dimensional Barnes-Rivers operators, namely [14], 


$$
\begin{aligned}
& P_{\mu \nu, \rho \sigma}^{1}=\frac{1}{2}\left(\theta_{\mu \rho} \omega_{\nu \sigma}+\theta_{\mu \sigma} \omega_{\nu \rho}+\theta_{\nu \rho} \omega_{\mu \sigma}+\theta_{\nu \sigma} \omega_{\mu \rho}\right), \\
& P_{\mu \nu, \rho \sigma}^{2}=\frac{1}{2}\left(\theta_{\mu \rho} \theta_{\nu \sigma}+\theta_{\mu \sigma} \theta_{\nu \rho}-\theta_{\mu \nu} \theta_{\rho \sigma}\right), \\
& P_{\mu \nu, \rho \sigma}^{0}=\frac{1}{2} \theta_{\mu \nu} \theta_{\rho \sigma}, \quad \bar{P}_{\mu \nu, \rho \sigma}^{0}=\omega_{\mu \nu} \omega_{\rho \sigma}, \\
& \overline{\bar{P}}_{\mu \nu, \rho \sigma}^{0}=\theta_{\mu \nu} \omega_{\rho \sigma}+\omega_{\mu \nu} \theta_{\rho \sigma},
\end{aligned}
$$

where $\theta_{\mu \nu}$ and $\omega_{\mu \nu}$ are the usual transverse and longitudinal vector projector operators $\theta_{\mu \nu}=\eta_{\mu \nu}-k_{\mu} k_{\nu} / k^{2}, \quad \omega_{\mu \nu}$ $=k_{\mu} k_{\nu} / k^{2}$, where $k_{\mu}$ is the momentum of the graviton exchanged, and the operator

$$
P_{\mu \nu, \rho \sigma} \equiv \frac{\square \partial^{\lambda}}{4}\left[\varepsilon_{\mu \lambda \rho} \theta_{\nu \sigma}+\varepsilon_{\mu \lambda \sigma} \theta_{\nu \rho}+\varepsilon_{\nu \lambda \rho} \theta_{\mu \sigma}+\varepsilon_{\nu \lambda \sigma} \theta_{\mu \rho}\right],
$$

which has its origin in the linearization of Eq. (3), i.e.,

$$
\mathcal{L}_{\mathrm{C} . \mathrm{S}_{\text {.lin }}}=\frac{1}{2} \frac{1}{M} h^{\mu \nu} P_{\mu \nu, \rho \sigma} h^{\rho \sigma},
$$

where $M \equiv \mu / \kappa^{2}$.

Linearizing $\mathcal{L}$ and adding to it the gauge-fixing Lagrangian

$$
\mathcal{L}_{\text {g.f. }}=-\lambda_{1} A_{\nu}^{2}-\frac{b}{4}\left[\lambda_{2}\left(A^{\mu}, \mu\right)^{2}+\lambda_{3} F_{\mu \nu}^{2}\right]
$$

where $\lambda_{1}, \lambda_{2}$ and $\lambda_{3}$ are gauge parameters, $A^{\mu} \equiv h^{\mu \nu}{ }_{, \nu}$, $F_{\mu \nu}=A_{\mu, \nu}-A_{\nu, \mu}$ and $b \equiv \beta \kappa^{2} / 2$, which corresponds to the Julve-Tonin gauge [15], we obtain a Lagrangian $\overline{\mathcal{L}}$ which can be written as $\overline{\mathcal{L}}=h^{\mu \nu} \overline{\mathcal{O}}_{\mu \nu, \rho \sigma} h^{\rho \sigma}$. Expanding now the operator $\overline{\mathcal{O}}$ in the basis $\left\{P^{1}, P^{2}, P^{0}, \bar{P}^{0}, \overline{\bar{P}}^{0}, P\right\}$ we get

$$
\begin{aligned}
\overline{\mathcal{O}}= & \left(-\lambda_{1} k^{2}-\lambda_{3} \frac{1}{2} b k^{4}\right) P^{1}+\left(k^{4} \frac{1}{2} b-k^{2}\right) P^{2}+\left(k^{3}+\frac{3}{2} b k^{4}\right. \\
& \left.+4 k^{4} b c\right) P^{0}+\left(-k^{4} \frac{1}{2} b \lambda_{2}-2 \lambda_{1} k^{2}\right) \bar{P}^{0}+P / M,
\end{aligned}
$$

where $c \equiv \alpha / \beta$.

The propagator is then given by

$$
\begin{aligned}
\mathcal{O}= & \frac{-2}{k^{2}\left[2 \lambda_{1}+b \lambda_{3} k^{2}\right]} P^{1}+\left[-\frac{1}{k^{2}}+\frac{1}{1+\frac{b M_{2}^{2}}{2}} \frac{1}{k^{2}-M_{2}^{2}}\right. \\
& \left.+\frac{1}{1+\frac{b M_{1}^{2}}{2}} \frac{1}{k^{2}-M_{1}^{2}}\right] P^{2}+\left[\frac{1}{k^{2}}-\frac{1}{k^{2}-m^{2}}\right] P^{0} \\
& -\frac{1}{k^{2}\left[2 \lambda_{1}+\lambda_{2} \frac{b}{2} k^{2}\right]} \bar{P}^{0}-\left[\frac { 4 } { b ^ { 2 } M ( M _ { 1 } ^ { 2 } - M _ { 2 } ^ { 2 } ) } \left(\frac{1}{k^{2}-M_{1}^{2}}\right.\right. \\
& \left.\left.-\frac{1}{k^{2}-M_{2}^{2}}\right) \frac{1}{k^{4}}\right] P
\end{aligned}
$$

where

$$
\begin{aligned}
& M_{1}^{2} \equiv\left(\frac{2}{b^{2} M^{2}}\right)\left[1+b M^{2}+\sqrt{1+2 b M^{2}}\right], \\
& M_{2}^{2} \equiv\left(\frac{2}{b^{2} M^{2}}\right)\left[1+b M^{2}-\sqrt{1+2 b M^{2}}\right], \\
& m^{2} \equiv \frac{-1}{b\left(\frac{3}{2}+4 c\right)} .
\end{aligned}
$$

If we do not want tachyons in the dynamical field, we may choose, for instance, $b>0$ and $\left(\frac{3}{2}+4 c\right)<0$. In this case the theory is causal at the tree level. We assume that this is the case from now on.

Let us then expand the sources in a suitable basis. The set of independent vectors in momentum space,

$$
k^{\mu} \equiv\left(k^{0}, \mathbf{k}\right), \quad \tilde{k}^{\mu} \equiv\left(k^{0},-\mathbf{k}\right), \quad \varepsilon^{\mu} \equiv(0, \vec{\epsilon}),
$$

where $\vec{\epsilon}$ is a unit vector orthogonal to $\mathbf{k}$, serves our purpose. As a consequence the symmetric current tensor $T^{\mu \nu}(k)$ can be written as

$T^{\mu \nu}=a k^{\mu} k^{\nu}+b \widetilde{k}^{\mu} \widetilde{k}^{\nu}+c \varepsilon^{\mu} \varepsilon^{\nu}+d k^{(\mu} \widetilde{k}^{\nu)}+e k^{(\mu} \varepsilon^{\nu)}+f \widetilde{k}^{(\mu} \varepsilon^{\nu)}$.

The current conservation, $k_{\mu} T^{\mu \nu}=0$, gives the following constraints for the coefficients $a, b, d, e$ and $f$ :

$$
\begin{aligned}
a k^{2}+\left(k_{0}^{2}+\mathbf{k}^{2}\right) d / 2 & =0, \\
b\left(k_{0}^{2}+\mathbf{k}^{2}\right)+d k^{2} / 2 & =0 \\
e k^{2}+f\left(k_{0}^{2}+\mathbf{k}^{2}\right) & =0 .
\end{aligned}
$$

If we saturate the indices of $T^{\mu \nu}$ with $k_{\mu}$, we obtain the equation $k_{\mu} k_{\nu} T^{\mu \nu}=0$, which yields a consistency relation for the coefficients $a, b$ and $d$

$$
a k^{4}+b\left(k_{0}^{2}+\mathbf{k}^{2}\right)^{2}+d k^{2}\left(k_{0}^{2}+\mathbf{k}^{2}\right)=0 .
$$

Therefore, all we have to do now is to compute the residue of $\mathcal{A}$ at each first-order pole of the propagator and verify its sign. From Eqs. (4) and (6) we obtain at once

$$
\begin{aligned}
\mathcal{A}= & g^{2}\left[\frac{2}{b^{2}} \frac{b M_{2}^{2}-2}{\left(M_{2}^{2}-M_{1}^{2}\right) M_{2}^{2}} \frac{T_{\mu \nu} T^{\mu \nu}-\frac{1}{2} T^{2}}{k^{2}-M_{2}^{2}}\right. \\
& +\frac{2}{b^{2}} \frac{2-b M_{1}^{2}}{\left(M_{2}^{2}-M_{1}^{2}\right) M_{1}^{2}} \frac{T_{\mu \nu} T^{\mu \nu}-\frac{1}{2} T^{2}}{k^{2}-M_{1}^{2}}-\frac{\frac{1}{2} T^{2}}{k^{2}-m^{2}} \\
& \left.+\frac{4}{b^{2} M_{1}^{2} M_{2}^{2}} \frac{T^{2}-T_{\mu \nu} T^{\mu \nu}}{k^{2}}\right] .
\end{aligned}
$$

Thus, $\left.\operatorname{Res} \mathcal{A}\right|_{k^{2}=M_{1}^{2}}>0,\left.\quad \operatorname{Res} \mathcal{A}\right|_{k^{2}=M_{2}^{2}}>0,\left.\quad \operatorname{Res} \mathcal{A}\right|_{k^{2}=m^{2}}$ $<0$ and $\left.\operatorname{Res} \mathcal{A}\right|_{k^{2}=0}=0$. The theory is causal and has one 
physical massless spin-2 particle, two physical spin-2 particles of masses equal to $M_{1}$ and $M_{2}$, respectively, and one spin- 0 ghost of mass $m$. The massless excitation, like the massless excitation of Einstein gravity in $(2+1) \mathrm{D}$, is not a dynamical degree of freedom, i.e., it is nonpropagating. Consequently, topological 3DHDG is nonunitary at the tree level.

\section{FINAL REMARKS}

We have shown that the addition of a nontrivial topological term to 3DHDG is not a good therapy to cure the nonunitarity of the latter. However, one can show that the theory possesses a well-behaved effective nonrelativistic potential.

To conclude we prove that the addition of a topological term to three-dimensional $R+R^{2}$ gravity-which is treelevel unitary-spoils the unitarity of the aforementioned theory. Topological three-dimensional $R+R^{2}$ gravity is defined by the Lagrangian

$$
\mathcal{L}=-\frac{2 R \sqrt{g}}{\kappa^{2}}+\frac{\alpha}{2} R^{2} \sqrt{g}+\mathcal{L}_{\text {C.S. }},
$$

where $\mathcal{L}_{\text {C.S. }}$ is given by Eq. (3).

Proceeding exactly as we have done in Sec. III, we get that the propagator in the Julve-Tonin gauge [15] as well as the current-current amplitude for the above can be written as

$$
\begin{aligned}
\mathcal{O}^{-1}= & \frac{-1}{\lambda_{1} k^{2}} P^{1}+\left[-\frac{1}{k^{2}}+\frac{1}{k^{2}-M^{2}}\right] P^{2}+\left[\frac{1}{k^{2}}-\frac{1}{k^{2}-m^{2}}\right] P^{0} \\
& -\frac{1}{2 \lambda_{1} k^{2}} \bar{P}^{0}+\left[\frac{M}{k^{2}-M^{2}} \frac{1}{k^{4}}\right] P, \\
\mathcal{A}= & \mathrm{g}^{2}\left[\frac{T_{\mu \nu} T^{\mu \nu}-\frac{1}{2} T^{2}}{k^{2}-M^{2}}-\frac{\frac{1}{2} T^{2}}{k^{2}-m^{2}}+\frac{T^{2}-T_{\mu \nu} T^{\mu \nu}}{k^{2}}\right],
\end{aligned}
$$

where $m^{2} \equiv-1 / 2 \alpha \kappa^{2}$.

Assuming thenceforth that $-\alpha>0$, which implies that there are no tachyons in the dynamical field, we promptly obtain that $\left.\operatorname{Res} \mathcal{A}\right|_{k^{2}=M^{2}}>0,\left.\quad \operatorname{Res} \mathcal{A}\right|_{k^{2}=m^{2}}<0 \quad$ and $\left.\operatorname{Res} \mathcal{A}\right|_{k^{2}=0}=0$. The theory is causal and has one normal particle of spin- 2 and mass $\mathrm{M}$, one spin- 0 ghost of mass $m$ and one normal massless spin-2 particle. The massless excitation is nonpropagating. Accordingly, topological threedimensional $R+R^{2}$ gravity is tree-level nonunitary.

Although being tree-level nonunitary, topological threedimensional $R+R^{2}$ gravity has a well-behaved nonrelativistic potential. In fact, using a procedure similar to that developed in Ref. [5], we find that the potential can be expressed as

$$
V(r)=2 \bar{m} \bar{G}\left[\kappa_{0}(r m)-\kappa_{0}(r M)\right],
$$

which behaves as $2 \bar{m} \bar{G} \ln (\mathrm{M} / \mathrm{m})$ at the origin and as

$$
2 \bar{G} \bar{m}\left[\sqrt{\frac{\pi}{2 m r}} e^{-m r}-\sqrt{\frac{\pi}{2 M r}} e^{-M r}\right]
$$

asymptotically, where $\bar{m}$ is the mass of the source that generates the gravitational field.

To conclude we call attention to the fact that the topological Chern-Simons term is not a panacea for 3DHDG's unitarity problem. Furthermore, the above is responsible for breaking down the tree-level unitarity of $R+R^{2}$ gravity in $(2+1) \mathrm{D}$. In other words, the coexistence between the topological Chern-Simons term and quadratic gravity theories in $(2+1) \mathrm{D}$ is conflicting.

\section{ACKNOWLEDGMENT}

The author is very grateful to CNPq-Brazil for financial support.
[1] S. Deser and R. Jackiw, Ann. Phys. (N.Y.) 152, 220 (1984); 153, 405 (1984).

[2] S. Deser, R. Jackiw, and S. Templeton, Phys. Rev. Lett. 48, 975 (1982); Ann. Phys. (N.Y.) 140, 372 (1982).

[3] A. Accioly, A. Azeredo, and H. Mukai, Phys. Lett. A 279, 169 (2001).

[4] A. Accioly, A. Azeredo, and H. Mukai, Mod. Phys. Lett. A 16, 1449 (2001).

[5] A. Accioly, H. Mukai, and A. Azeredo, Class. Quantum Grav. 18, L31 (2001).

[6] S. Deser and Z. Yang, Class. Quantum Grav. 7, 1603 (1990).

[7] C. Pinheiro, G. Pires, and C. Sasaki, Gen. Relativ. Gravit. 29,
409 (1997).

[8] P. Teyssandier, Class. Quantum Grav. 6, 219 (1989).

[9] A. Accioly et al., Prog. Theor. Phys. 104, 103 (2000).

[10] B. Linet and P. Teyssandier, Class. Quantum Grav. 9, 159 (1992).

[11] S. Deser, Phys. Rev. Lett. 64, 611 (1990).

[12] S. Giddikgs, J. Abbott, and K. Kuchăr, Gen. Relativ. Gravit. 16, 751 (1984).

[13] P. van Nieuwenhuizen, Nucl. Phys. B60, 478 (1973).

[14] I. Antoniadis and E. Tomboulis, Phys. Rev. D 33, 2756 (1986).

[15] J. Julve and M. Tonin, Nuovo Cimento Soc. Ital. Fis., B 46, 137 (1987) 